\title{
Layout Analysis And Optimization Of Assembly Workshop Of Bogie
}

\author{
Lei Tang ${ }^{1, a}$, Li Sun ${ }^{1, a}$ and Jiang Wei ${ }^{2, b}$ \\ ${ }^{1}$ School of mechanical engineering, Dalian Jiaotong University, Dalian 116028, China. \\ ${ }^{2}$ Zhongche group, Daian Locomotive Vehicle Co Ltd, Dalian 116023, China. \\ atanglei112211@163.com
}

Keywords: bogie, layout analysis, simulation, em-plant.

\begin{abstract}
. with the fast rapid development of high-speed railway and heavy haul, the company of a locomotive bogie assembly line production capacity and production efficiency needs to be improved. In order to improve the production efficiency of the assembly workshop of the bogie and improve annual output and save the cost, the present paper makes an investigation and Analysis on the present situation of the workshop layout. Using the simulation software em-Plant to build the simulation model to analyze the data, provides reliable basis for further optimization of the parameters of the layout.
\end{abstract}

\section{Introduction}

With the rapid development of rail transportation equipment manufacturing technology and continuous increase of market demand, in order to constantly improve the production capacity, to meet the market demand, a locomotive works is facing the problem of expansion and the transferring. In order to achieve a higher production capacity, in the case of the original factory cannot increase, enterprises have to move. To implement the lean process management as the key link to ensure the new factory "high starting point, high goals, high quality, high efficiency, high benefit" of the planning and construction.

Production line layout planning is one of the most important one annulus. The content and meaning of this study is that through the rational planning of the production line layout, how to improve the plant production efficiency, resource utilization, economic efficiency and reduce production cost. The reasonable equipment layout can ensure the fluency of material handling in the workshop, improve the utilization rate of the workshop space effectively, and provide a healthy and comfortable working environment for operating personnel. Therefore, equipment layout design and evaluation are required, whether to establish a new workshop, or to rearrange the original workshop. In the assembly production line of a locomotive works, it is inevitable that there are some unreasonable procedure arrangement, such as long production cycle, personnel no working time, low utilization rate of equipment. It can adopt the method of computer simulation, using the system simulation software, to seek the solution.

For drive unit and bogie assembly line production status in a locomotive works, we can choose the em-Plant software in modeling and simulation, and on the basis of ensuring the equipment utilization rate, by compressing the beat, reached the production line balancing effect, thereby improving the bogie assembly line production.

\section{To establish the simulation model of the old layout}

Nowadays, manufacturing system is a set of modern machinery manufacturing, computer science and engineering management in an integrated application, due to its technical complexity, huge investment, the construction entity system research is clearly unreasonable. So in the design stage of the manufacturing system , through the simulation system can be selected the best structure and configuration scheme, to ensure that the system can accomplish the design requirements and can get very good economy, flexibility and reliability, and can effectively prevent the larger economic loss; 
In the stage of the manufacturing process, through the simulation can predict the performance of the system under different scheduling, in order to determine reasonable and effective operation plan, find out the "bottleneck" link in the system, which can give full play to the production capacity of manufacturing systems, improve the economic benefit.

In the study of layout design and optimization, we can use tools of modeling and simulation, to obtain the actual guidance of the actual production of the layout model and production planning and other objectives.

The following figure is bogie drive unit assembly flow chart, according to the flow chart, control layout, simulation model can be established intuitively.

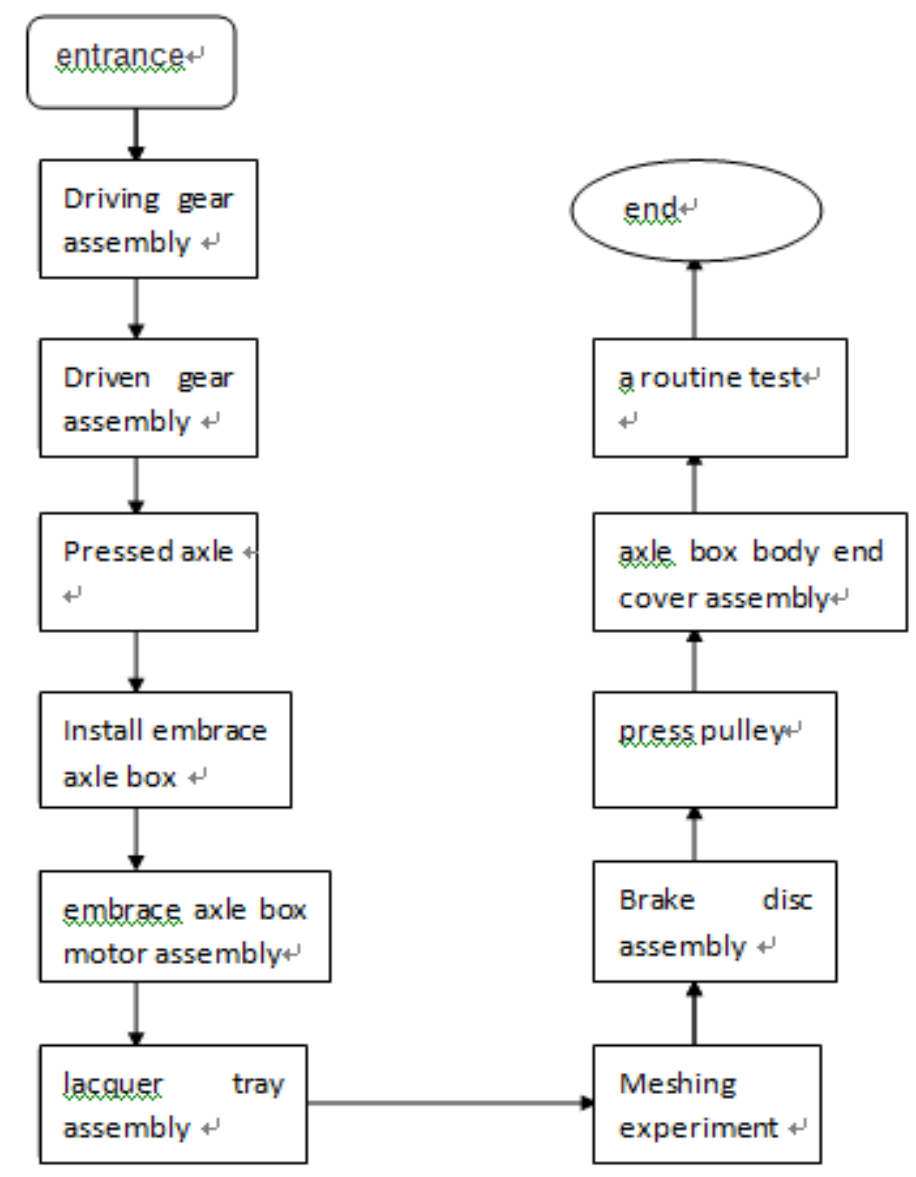

Fig. 1 HXD3B type drive unit assembly flow chart

Running software can output in figure 2, figure 3, to analyze the output chart, can be seen from the following two figures driving gear assembly etc. six processes are all half an hour, suggesting that these same equipment working time is shorter, the rest of the time in the blocked state. Why this happens, because the current processing is completed, the item will get to the next assembly processing, but the present processing is slow, before items will be waiting for the back of the processing is completed, this is the jam, equipment is in a state of not working for a long time, the impact on the benefit of the factory will, test bed utilization rate is very high, as the bottleneck process, the processing time is longer, the other equipment utilization rate is low, reaction to the imbalance of the assembly line. 


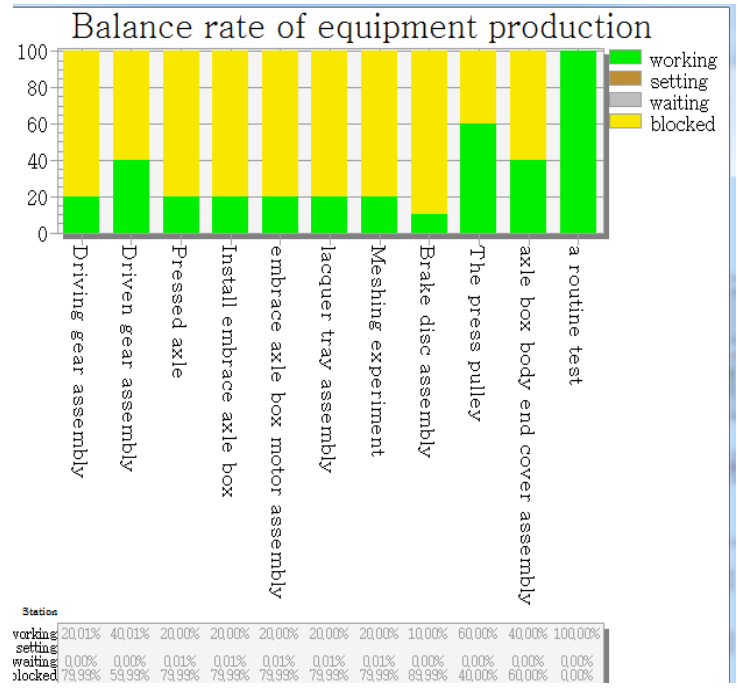

Fig.2 Equipment utilization rate

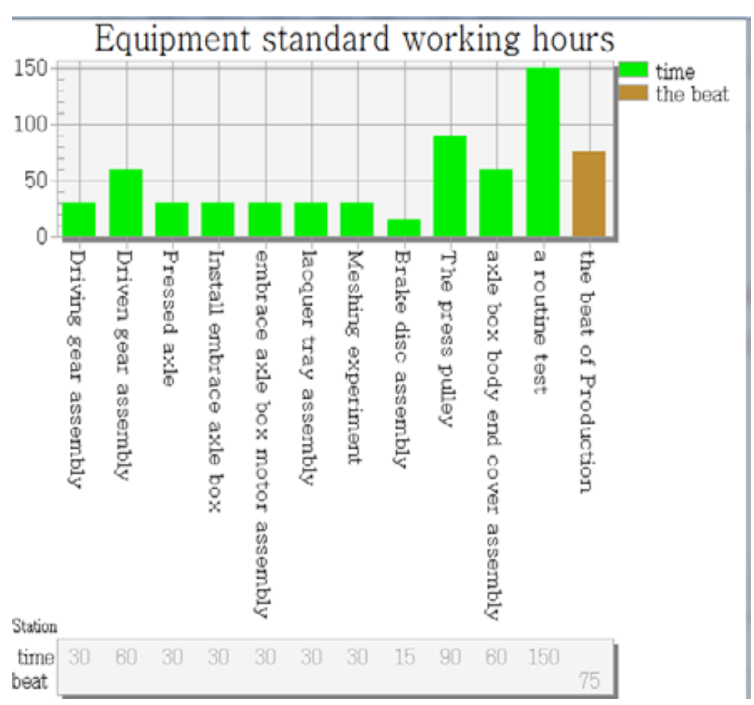

Fig.3 Devices work time

Here to do a test, the clock in this object, we set running time one year, because the platform with shifts in the placement of an object, so the operation of all equipment meet the locomotive plant idle time to rest. Click on running button.

To open the last process station, which is namely the object of routine test bench, in the column attribute, the statistics can be seen that into number is 1474, exit number is 1473 . Because it is the last station, so exit number is the number of finished drive unit. 1473 units is an annual output.

In the process of research, It can be known that the locomotive works need to achieve an annual output of 500 sets. Each bogie has six drive unit, also is the capacity of drive unit for 3000, and our production line output is 1473 , is nearly $50 \%$, far did not reach the target, which is also fits with the above image. Achieve production minimum production rhythm is 75 minutes, and the wheel pressure machine and routine test bench are beyond the production rhythm, it also demonstrates the authenticity of the simulation.

\section{Layout Optimization}

In the daily business of manufacturing, It will usually encounter layout optimization problem, and the layout optimization for enterprise represents the benefit represents productivity, so occupies the important position. The following is the maximum productivity, the lowest cost, maximum profit, 3 aspects of optimization.

(1) the highest productivity index

It refers to the time needed for manufacturing unit product at least, or the number of products produced in unit time. When on a certain product is an urgent need to, often using the index, while the costs and profits as a secondary factor.

(2) the lowest cost index

It refers to a single product of the lowest cost. When the time no special requirements to the output of products, adopt this measure can make the product with the biggest competitiveness.

(3) the largest profit index

It refers to the time the largest profit. At this time do not pursue the lowest cost, through increased investment in production to reduce the unit production time, so that the production and sales more products, gain more profits.

Defined by the above you can see the highest productivity index under the production of a single component the shortest cycle time, use the lowest cost index, the longest cycle time, the maximum profit targets, between the two.

2.1 the optimization of the highest productivity

Below the chart is not limit equipment investment and to increase the number of multiple bottleneck process equipment of the ideal state. Shown below equipment utilization rate is very high, no bottleneck process, finally it is concluded that producing $\mathrm{P}$ balance rate is as high as $95.4 \%$. 


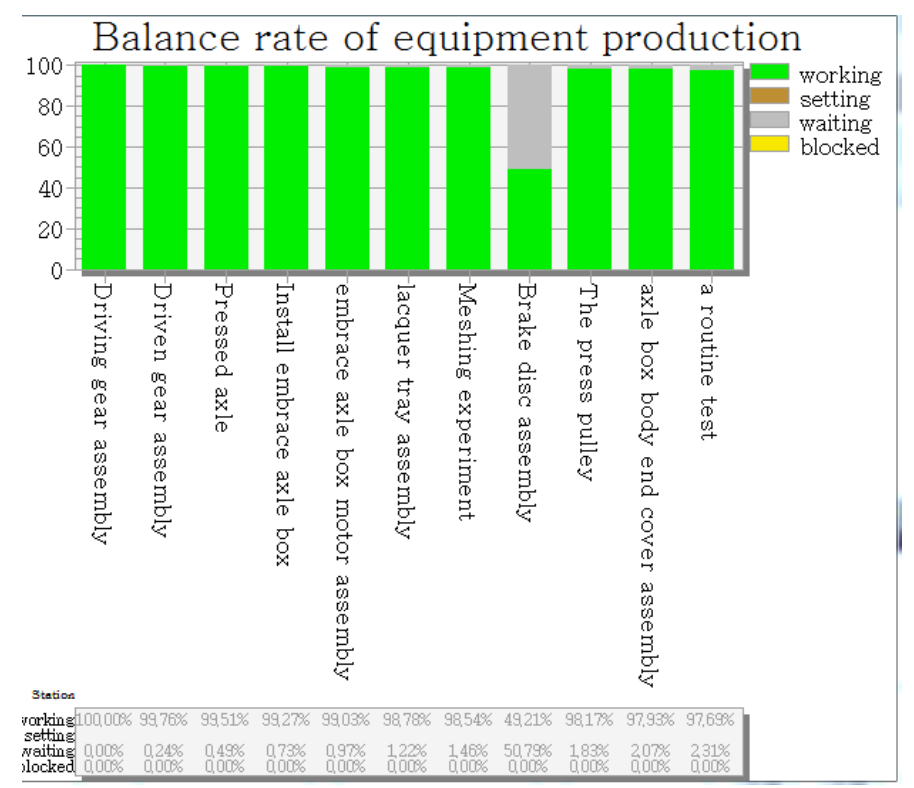

Fig. 4 Balance rate of highest productivity cases

The following is a simulation software simulation running, and concluded that the annual output.

Joined in the simulation model called ShiftCalendar module, the function of it is on the workshop production of time constraints, such as workers 2 shifts a day job, and working day from Monday to Friday, including legal holiday break.

It can achieve the data that through the biggest productivity layout optimization, brinkley drive unit of the annual output of 7375 units. Of course in order to continue to increase production can also implement frequency, lengthen working hours, etc. System measures.

2.2 the lowest cost optimization

Cases, optimize the chart below is the lowest cost, in the case of the lowest cost of each working procedure is only one same to carry on the processing and assembling, so in this case the equipment cost is very low. Because each device need workers to operate assembly, so at the same time reduce the labor costs. Finally it is concluded that production balance rate $\mathrm{P}$ was $33.6 \%$.

The lowest cost cases balance rate as shown in figure 2 .

The following is a simulation software simulation run, and concluded that the annual output. Of course it also joined the ShiftCalendar module. The workers were still for 2 classes a day, of course, in order to compress the cost can be reduced to one a day.

The minimum annual cost conditions simulation output is 1473 .

2.3 optimization of maximum profit

Below cases, optimize the chart is the biggest profit, under the condition of maximum profit in the driven gear assembly procedure and add a same axle box body cover installation process to carry on the processing and assembling, the two processes of equipment investment cost is not much, but also increase a same enough to meet the optimization.

The third increase in the same process as the routine experiment process, because of this process area is larger, the overall layout for the factory, only add a same. Process and the press pulley wheel pressure machine for import of used machines, higher cost, cover an area of an area is not small, so if you add the same, is not in conformity with the largest profit optimization.

Finally it is concluded that production balance rate $\mathrm{P}$ was $42.4 \%$.

The following is a simulation software simulation run, and concluded that the annual output. Of course it also joined the ShiftCalendar module. 


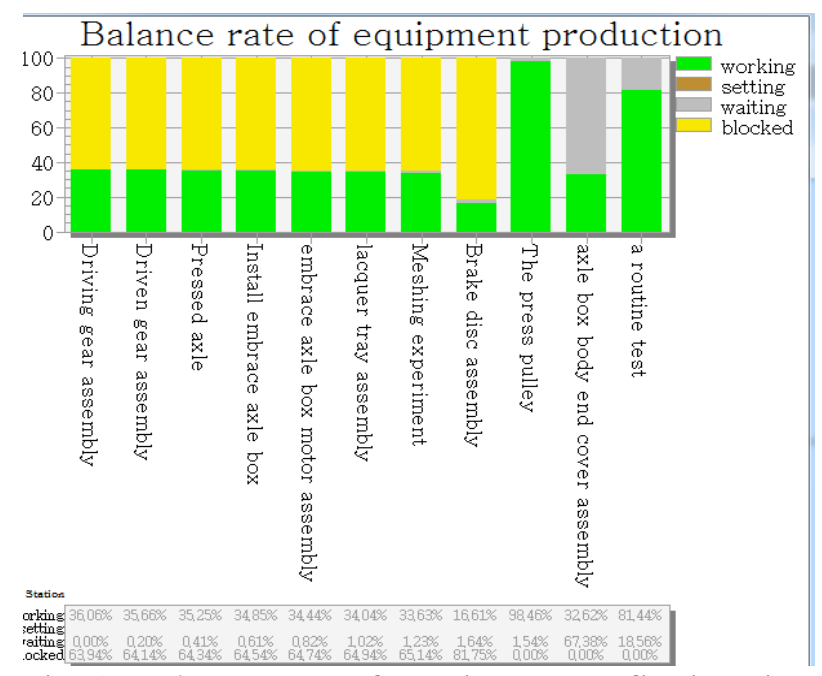

Fig.5 Balance rate of maximum profit situation

\section{Summary}

Based on em - plant platform of bogie of assembly production line are discussed and the research, through the analysis of the process flow of drive unit, corresponding to the layout of workshop and related data obtained by the simulation of operation, on the basis of the data is analyzed and the research, on the simulation platform, the measured data input, operation related chart data can be obtained, and the equipment real-time state, the analysis of the pros and cons of the whole process provides a powerful reference. To unreasonable place, from the highest productivity, minimum cost and maximum profit three aspects has been optimized, to improve, not only can satisfy the production problem, but also improve the locomotive plant efficiency, save the time.

\section{References}

[1] Li Sun, Jianmin Fan, Ziqin Ma, Xiulun Wang, Hao Tan, Aiqiao Sun, Tingting Xu. Product Life Cycle-oriented Cloud Process Management Research. Applied Mechanics and Materials,Trans Tech Publications, Switzerland, 2012,120:456-459

2] Zou Shiwei. Workshop flexible layout algorithm and three-dimensional simulation study [D]. Wuhan: wuhan university of technology. 2007.

[3] Sun Honghua. Many varieties of medium batch machinery manufacturing workshop equipment layout optimization research [D]. Heilongjiang province, Harbin industrial university, 2008.

[4] week hero Korea, heterogenous, wu, etc. The distributed virtual manufacturing system application research [J]. Journal of tsinghua university, 2002, and (9) : 1265-1268.

[5] Zhu Weifeng. Complex logistics system simulation and its status quo [J]. Journal of system simulation, 2003, 15 (3) : 353-356.

[6] P.S.Welgama and P.R.Gibson. A construction algorithm for the machine layout with fixed pick-up and drop-off point [J]. International Journal of production research, 1993, 11(31): $2575-2590$. 